\title{
The intertwisted correlations among non-alcoholic fatty liver disease, atherosclerosis, and metabolic syndrome
}

\author{
Haruhisa Nakao • Masashi Yoneda
}

Published online: 3 October 2009

(C) Springer 2009

Non-alcoholic fatty liver disease (NAFLD) refers to a spectrum of disorders from simple hepatic steatosis to nonalcoholic steatohepatitis (NASH), steatohepatitis with inflammation and fibrosis, which can progress to cirrhosis, and hepatocellular carcinoma. NAFLD is the most common cause of abnormal liver function tests. The prevalence of NAFLD is approximately between 10 and $30 \%$ of the general population in various countries and considered to be increasing [1-3]. Previous studies have shown that NAFLD is strongly associated with obesity, type 2 diabetes mellitus, dyslipidemia and insulin resistance, which are all features of the metabolic syndrome [4, 5]. Accordingly, NAFLD is considered the hepatic manifestation of the metabolic syndrome $[6,7]$. The metabolic syndrome is a risk factor for the development of cardiovascular disease and a predictor of type 2 diabetes mellitus [8-10]. A large number of studies have demonstrated that metabolic syndrome apparently associates with cardiovascular disease [11]. In view of several lines of evidence, NAFLD would relate to cardiovascular disease and might play a role in the development of atherosclerosis.

Recently, the importance of the association between NAFLD and cardiovascular disease has been focused, and several studies have revealed that NAFLD is related to carotid artery intima-media thickness (IMT), a marker of subclinical atherosclerosis, independent of insulin resistance, metabolic syndrome components, and the traditional

The online version of the article referred to in this editorial can be found under doi: 10.1007/s00535-009-0125-8.

H. Nakao $(\bowtie) \cdot$ M. Yoneda

Division of Gastroenterology, Department of Internal Medicine, Aichi Medical University, 21 Karimata Yazako, Nagakute,

Aichi 480-1195, Japan

e-mail: hnakao@aichi-med-u.ac.jp risk factors for cardiovascular disease [12, 13]. Carotid IMT, as measured noninvasively by ultrasonography, is directly associated with increased risk of cardiovascular disease. In addition, carotid IMT has been shown to be an independent predictor of cardiovascular disease after adjustment for traditional risk factors, and the only noninvasive imaging test recommended by the American Heart Association for inclusion in the evaluation of risk [14, 15]. Brea et al. [12] reported that patients with NAFLD have significantly greater carotid IMT than age and sex matched patients without NAFLD, independent of the classical risk factor of the metabolic syndrome, in a hospital-based casecontrol study. NAFLD is suggested to be a strong risk factor for carotid atherosclerosis beyond its association with metabolic syndrome. Furthermore, Targher et al. [16] showed that NAFLD is independently associated with an increased incidence of cardiovascular events in type 2 diabetic patients. Interestingly, the severity of liver histopathology among NAFLD patients is strongly associated with early carotid atherosclerosis, independent of classical risk factors and the presence of metabolic syndrome [17]. The relation between NAFLD and carotid IMT was investigated by several hospital-based or population-based case control studies [18]. However, some studies examined the association in a small population, or utilized mainly diabetic individuals, although a German study investigated a random large population sample [19].

In this issue of the Journal of Gastroenterology, Carallo et al. [20] studied a large population mainly consisting of nondiabetic patients but with cardiovascular or metabolic risk factors in a hospital-based case-control study, and demonstrated that hepatic steatosis was associated with cardio atherosclerosis, independent of metabolic syndrome, cardiovascular diseases, diabetes mellitus, and/or insulin resistance, as well as previous reports in diabetic patients. 
In addition, their study, the STEATO study, also showed that HOMA index was not associated with carotid atherosclerosis despite of the association between the metabolic syndrome and carotid atherosclerosis, and carotid atherosclerosis was not associated with aspartate aminotransferase (AST) and alanine aminotransferase (ALT) but with gamma-glutamyl transpeptidase (GGT).

Serum ALT levels are a serological marker of liver inflammation or injury, and serum GGT levels are a diagnostic tool for hepatobiliary disorder and alcohol intake. In general, ALT is insensitive for the detection of NAFLD and cannot be reliably used to exclude the presence of advanced liver disease, because ALT may be normal in up to $78 \%$ of patients with NAFLD and the histological spectrum of disease may be present among patients with normal ALT levels, while GGT levels may be above the normal range in some patients with NAFLD [4]. Several previous studies demonstrated that GGT levels, even when within the normal range, correlate with the presence of several components of the metabolic syndrome [21]. Recently, ALT and GGT have received considerable attention because several studies have suggested that elevated serum ALT and GGT increases the risk of carotid atherosclerosis in patients with NAFLD and might serve as a surrogate marker of cardiovascular risk and metabolic syndrome. In particular, a number of studies support the importance of GGT with regard to the association with cardiovascular disease since GGT has a stronger, more specific association with metabolic syndrome and atherosclerosis than ALT. The STEATO study showed that the prevalence of subjects with hepatic steatosis and normal level of aminotransferases, i.e., ALT and AST was almost $60 \%$ and concluded that transaminase activity might not predict cardiovascular disease. In contrast, several investigators have shown that serum GGT activity predicts future development of not only metabolic syndrome but also cardiovascular disease [22]. However, serum GGT levels cannot predict increased risk of cardiovascular disease in every study. A recent large population-based cohort study indicated that elevated GGT was associated with mortality from all causes, liver disease, and diabetes, but not from cardiovascular disease, while ALT was not associated with all-cause mortality but only with liver disease mortality [23]. Further large cohort studies with long-term follow up will be necessary to determine the impact of GGT on the mortality from cardiovascular disease in patients with NAFLD.

NAFLD is the most common cause of liver disease not only in adults but also in children. Recently, Pacifico et al. [24] investigated obese children to assess whether NAFLD in children is associated with increased carotid IMT. Surprisingly, obese children with NAFLD had significantly increased carotid IMT compared to obese children without liver involvement and control children. It is important to note that NAFLD is strongly associated with carotid atherosclerosis even in childhood. Schwimmer et al. [25] performed a case control study in a large clinical sample of obese children and adolescents with and without NAFLD and showed that in obese children, NAFLD is strongly associated with multiple cardiovascular risk factors. They suggested that the identification of NAFLD in a child should prompt consideration of cardiovascular health, and the therapeutic goals for NAFLD should include not only the prevention of end-stage liver disease but also cardiovascular disease and diabetes. On the basis of this evidence, some investigators speculated that fatty liver is a risk factor for early onset of atherosclerosis that may progress in adulthood [3].

NAFLD, another feature of the metabolic syndrome, is associated with increased cardiovascular disease risk, independent of the metabolic syndrome components and traditional risk factors of atherosclerosis. Although the exact mechanism by which NAFLD contributes to the risk of atherosclerosis remains obscure, several hypotheses have been proposed [3, 12]. Some researchers have assumed that NAFLD and atherosclerosis might share common mediators, while others have hypothesized that NAFLD itself might play an important role in the progression of atherosclerosis. Another possibility is that NAFLD might contribute to increased insulin resistance and dyslipidemia or reduced levels of adiponectin so that atherosclerosis might be accelerated. Another possible mechanism is that increased oxidative stress and underlying inflammation might have an effect on the association between NAFLD and atherosclerosis $[26,27]$. Further investigations are needed to clarify the mechanism to explain the association between NAFLD and atherosclerosis.

Finally, NAFLD is a strong risk factor for carotid atherosclerosis beyond its association with metabolic syndrome. It is important to note that the detection of hepatic steatosis should alert us to the possible existence of underlying cardiovascular risk factors and therapeutic goals for NAFLD should include the prevention of cardiovascular disease as well as the prevention of advanced liver disease.

\section{References}

1. Angulo P. Nonalcoholic fatty liver disease. $\mathrm{N}$ Engl $\mathrm{J}$ Med. 2002;346:1221-31.

2. Williams R. Global challenges in liver disease. Hepatology. 2006;44:521-6.

3. Targher G. Non-alcoholic fatty liver disease, the metabolic syndrome and the risk of cardiovascular disease: the plot thickens. Diabet Med. 2007;24:1-6. 
4. Adams LA, Angulo P. Recent concepts in non-alcoholic fatty liver disease. Diabet Med. 2005;22:1129-33.

5. Hamaguchi M, Kojima T, Takeda N, Nakagawa T, Taniguchi H, Fujii K, et al. The metabolic syndrome as a predictor of nonalcoholic fatty liver disease. Ann Intern Med. 2005;143:722-8.

6. Marchesini G, Brizi M, Bianchi G, Tomassetti S, Bugianesi E, Lenzi M, et al. Nonalcoholic fatty liver disease: a feature of the metabolic syndrome. Diabetes. 2001;50:1844-50.

7. Marchesini G, Bugianesi E, Forlani G, Cerrelli F, Lenzi M, Manini R, et al. Nonalcoholic fatty liver, steatohepatitis, and the metabolic syndrome. Hepatology. 2003;37:917-23.

8. Bonora E, Targher G, Formentini G, Calcaterra F, Lombardi S, Marini F, et al. The metabolic syndrome is an independent predictor of cardiovascular disease in type 2 diabetic subjects. Prospective data from the Verona Diabetes Complications Study. Diabet Med. 2004;21:52-8.

9. Neuschwander-Tetri BA. Nonalcoholic steatohepatitis and the metabolic syndrome. Am J Med Sci. 2005;330:326-35.

10. Marchesini G, Marzocchi R. Metabolic syndrome and NASH. Clin Liver Dis. 2007;11:105-17.

11. Qiao Q, Gao W, Zhang L, Nyamdorj R, Tuomilehto J. Metabolic syndrome and cardiovascular disease. Ann Clin Biochem. 2007;44:232-63.

12. Brea A, Mosquera D, Martin E, Arizti A, Cordero JL, Ros E. Nonalcoholic fatty liver disease is associated with carotid atherosclerosis: a case-control study. Arterioscler Thromb Vasc Biol. 2005;25:1045-50.

13. Targher G, Bertolini L, Poli F, Rodella S, Scala L, Tessari R, et al. Nonalcoholic fatty liver disease and risk of future cardiovascular events among type 2 diabetic patients. Diabetes. 2005;54:3541-6.

14. O'Leary DH, Polak JF. Intima-media thickness: a tool for artherosclerosis imaging and event prediction. Am J Cardiol. 2002;90:18-21.

15. Lorenz MW, Markus HS, Bots ML, Rosvall M, Sitzer M. Prediction of clinical cardiovascular events with carotid intima-media thickness: a systematic review and meta-analysis. Circulation 2007; 115:459-67.

16. Targher G, Bertolini L, Rodella S, Tessari R, Zenari L, Lippi G, et al. Nonalcoholic fatty liver disease is independently associated with an increased incidence of cardiovascular events in type 2 diabetic patients. Diabetes Care. 2007;30:2119-21.
17. Targher G, Bertolini L, Padovani R, Rodella S, Zoppini G, Zenari $\mathrm{L}$, et al. Relations between carotid artery wall thickness and liver histology in subjects with nonalcoholic fatty liver disease. Diabetes Care. 2006;29:1325-30.

18. Sookoian S, Prirola C. Non-alcoholic fatty liver disease is strongly associated with carotid atherosclerosis: a systematic review. J Hepatol. 2008;49:600-7.

19. Volzke H, Robinson DM, Kleine V, Deutscer R, Hoffmann W, Ludemann $\mathrm{J}$, et al. Hepatic steatosis is associated with an increased risk of carotid atherosclerosis. World J Gastroenterol. 2005; $11: 1848-53$.

20. Carallo C, Mancuso G, Mauro G, Laghi F, Madafferi B, Irace C. Hepatic steatosis, carotid atherosclerosis and metabolic syndrome: the STEATO study. J Gastoroenterol. 2009;44:1156-1161.

21. Kim DJ, Noh JH, Cho NH, Lee BW, Choi YH, Jung JH, et al. Serum gamma-glutamyltransferase within its normal concentration range is related to the presence of diabetes and cardiovascular risk factors. Diabet Med. 2005;22:1134-40.

22. Turgut O, Yilmaz A, Yalta K, Karadas F, Birhan YM. gammaGlutamyltransferase is a promising biomarker for cardiovascular risk. Med Hypotheses. 2006;67:1060-4.

23. Ruhl CE, Everhart JE. Elevated serum alanine aminotransferase and gamma-glutamyltransferase and mortality in the United States population. Gastroenterology. 2009;136:477-85.

24. Pacifico L, Cantisani V, Ricci P, Osborn JH, Schiavo E, Anania $\mathrm{C}$, et al. Nonalcoholic fatty liver disease and carotid atherosclerosis in children. Pediatr Res. 2008;63:423-7.

25. Schwimmer JB, Pardee PE, Lavine JE, Blumkin AK, Cook S. Cardiovascular risk factors and the metabolic syndrome in pediatric nonalcoholic fatty liver disease. Circulation. 2008;118:277-83.

26. Targher G, Bertolini L, Scala L, Zoppini G, Zenari L, Falezza G. Non-alcoholic hepatic steatosis and its relation to increased plasma biomarkers of inflammation and endothelial dysfunction in non-diabetic men. Role of visceral adipose tissue. Diabet Med. 2005;22:1354-8.

27. Yesilova Z, Yaman H, Oktenli C, Ozcan A, Uygun A, Cakir E, et al. Systemic markers of lipid peroxidation and antioxidants in patients with nonalcoholic fatty liver disease. Am J Gastroenterol. $2005 ; 100: 850-5$. 